\title{
XEROGEL $p$-ANISIDINAPROPILSÍLICA. ESTUDO DA ESTABILIDADE TÉRMICA E DA RESISTÊNCIA À LIXIVIAÇÃO COM SOLVENTES
}

Leonardo Franken, Lisiane S. dos Santos, Elina B. Caramão, Tania M. H. Costa e Edilson V. Benvenutti*

Instituto de Química, Universidade Federal do Rio Grande do Sul, CP 15003, 91501-970 Porto Alegre - RS

Recebido em 21/6/01; aceito em 30/10/01

\begin{abstract}
p-ANISIDINEPROPYLSILICA XEROGEL. THERMAL STABILITY AND RESISTANCE TO LEACHING BY SOLVENTS. The xerogel $p$-anisidinepropylsilica was obtained. This solid presents some residual paraffin and also a small fraction of high organofunctionalized material that was leached in polar solvent. The xerogel purification could be achieved by exhaustively washing with hexane and dichloromethane solvents, or submitting the xerogel to thermal treatment up to $300{ }^{\circ} \mathrm{C}$, in vacuum. The resulting purified xerogel material present an appreciable thermal stability and resistance to leaching by solvents.
\end{abstract}

Keywords: $p$-anisidine; infrared; stationary phase; sol-gel; thermal stability.

\section{INTRODUÇÃO}

A utilização de sílicas funcionalizadas com grupos orgânicos imobilizados na sua superfície tem recebido grande destaque no meio científico na última década, principalmente como materiais adorventes em processos de extração em fase sólida (SPE) e também como fase estacionária em cromatografia líquida $(H P L C)^{1-3}$. O grande interesse nesses materiais deve-se as suas propriedades finais. Esses materiais apresentam as características físicas do suporte sílica usado, enquanto suas propriedades químicas são devidas a fase orgânica presente na superfície. Dessa forma as propriedades químicas dos materiais podem ser facilmente monitoradas a partir da escolha do grupo orgânico a ser imobilizado.

Um método bastante utilizado na imobilização de grupos orgânicos sobre sílica é o enxerto ${ }^{1,4-6}$. Nesse método é comum usar-se um organosilano de fórmula $\mathrm{R}-\mathrm{Si}\left(\mathrm{OCH}_{3}\right)_{3}$, sendo $\mathrm{R}$ um grupo orgânico alifático, aromático ou ainda cadeia orgânica contendo outros grupos funcionais.

Outra possibilidade de se obter sílica organofuncionalizada é usar o método sol-gel ${ }^{7-11}$. Nesse método de síntese tem-se a formação de uma suspensão coloidal ( $\mathrm{sol}$ ) que através do processo de policondensação forma uma matriz sólida (gel). Após secagem total, sob condições brandas, esse gel é chamado de xerogel ${ }^{12-14}$. Organosilanos também são usados como precursores em reação de policondensação, juntamente com tetraetilortosilicato (TEOS) ou tetrametilortosilicato (TMSO).

O processo sol-gel tem vantagens sobre o método de enxerto, como por exemplo sua versatilidade na obtenção de materiais com propriedades físico-químicas distintas tais como área superficial, tamanho e forma de partículas, tamanho de poros, grau de incorporação orgânica, além do baixo custo e da simplicidade nos procedimentos experimentais ${ }^{15}$.

Recentemente demonstramos que é possível obter-se um xerogel de sílica contendo $p$-anisidina com diferentes graus de funcionalização orgânica ${ }^{16}$. Dando continuidade a esse estudo, é apresentado nesse trabalho a verificação da estabilidade térmica e a resistência à lixiviação com solventes desse xerogel. Assim é possível avaliar o seu potencial para futura utilização como material adsorvente em

\footnotetext{
*e-mail: edilson@iq.ufrgs.br
}

processos de extração em fase sólida ou como fase estacionária para colunas cromatográficas.

\section{PARTE EXPERIMENTAL}

\section{Síntese da $p$-anisidinapropilsílica ${ }^{16}$}

A $p$-anisidina foi inicialmente ativada por 30 minutos, com hidreto de sódio (60\% disperso em parafina) usando-se a mistura de solventes apróticos, hexano $(6.5 \mathrm{~mL})$ e THF $(6.5 \mathrm{~mL})$. Posteriormente foi adicionado $\left(\mathrm{CH}_{3} \mathrm{O}\right)_{3} \mathrm{Si}\left(\mathrm{CH}_{2}\right)_{3} \mathrm{Cl}$ (CPTMS). As quantidades de panisidina, NaH e CPTMS foram estequiométricas na concentração 0,35 mol. $\mathrm{L}^{-1}$. A mistura foi agitada por 3 horas sob argônio na temperatura de refluxo. $\mathrm{O}$ produto da reação $p$-anisidinapropiltrimetoxisilano (AnPTMS) foi então usado como precursor orgânico do xerogel $(10 \mathrm{~mL})$. A etapa de síntese do AnPTMS pode ser resumidamente descrita pela Eq 1 .

$$
\begin{aligned}
&\left(\mathrm{CH}_{3} \mathrm{O}\right)_{3} \mathrm{Si}\left(\mathrm{CH}_{2}\right)_{3} \mathrm{Cl}+\mathrm{Na}^{+-} \mathrm{NH}\left(\mathrm{C}_{6} \mathrm{H}_{6}\right) \mathrm{OCH}_{3} \rightarrow \\
&\left(\mathrm{CH}_{3} \mathrm{O}\right)_{3} \mathrm{Si}\left(\mathrm{CH}_{2}\right)_{3} \mathrm{NH}\left(\mathrm{C}_{6} \mathrm{H}_{6}\right) \mathrm{OCH}_{3}+\mathrm{NaCl}
\end{aligned}
$$

A essa solução foi adicionado sob agitação, $5 \mathrm{~mL}$ de etanol, $5 \mathrm{~mL}$ de tetraetilortosilicato (TEOS), $0,1 \mathrm{~mL}$ de $\mathrm{HF}$ (48\%) e água na razão estequiométrica com silício $\mathrm{r}=4 / 1(1,6 \mathrm{~mL})$. A condensação ocorre em $\mathrm{pH}=8$, através da catálise nucleofílica ${ }^{13,17}$. A mistura resultante foi guardada por 5 dias para gelificação, evaporação do solvente. $\mathrm{O}$ xerogel obtido foi triturado e secado em estufa a $100{ }^{\circ} \mathrm{C}$. Como resultado obteve-se um sólido violeta finamente dividido. A reação de condensação do xerogel pode ser descrita pela Eq. 2.

\section{Microscopia eletrônica de varredura (MEV)}

O xerogel foi analisado por microscopia eletrônica de varredura (MEV) com 20kV e 60000x de ampliação. A imagem foi processada usando-se o programa Quantikov ${ }^{18}$.

\section{Área superficial}

A área superficial do xerogel foi obtida pelo o método BET em um aparato volumétrico usando-se nitrogênio como molécula sonda. 


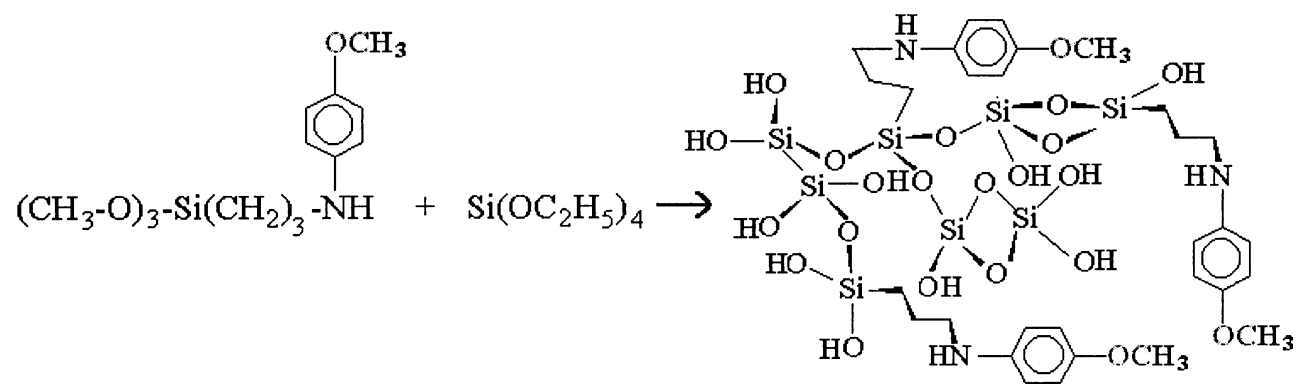

\section{Estabilidade térmica}

O estudo da estabilidade térmica da fase orgânica imobilizada, foi feito com o auxílio da espectroscopia no infravermelho, usandose uma cela de quartzo conectada a uma linha de vácuo. Esta cela tem um forno elétrico que permite submeter a amostra sólida a tratamento térmico, sob vácuo e possibilita a obtenção dos espectros no infravermelho sem expor a amostra a atmosfera externa ${ }^{19}$. O pó resultante da trituração do xerogel foi pressionado sob 1 ton $\mathrm{cm}^{-2}$. Os discos obtidos, de $100 \mathrm{mg}$ com $5 \mathrm{~cm}^{2}$ de área, foram aquecidos nas temperaturas de 100 a $450{ }^{\circ} \mathrm{C}$, sob vácuo $\left(10^{-3}\right.$ Torr $)$, durante $1 \mathrm{~h}$ para cada temperatura de análise. Os discos do material foram então analisados na região do infravermelho. O equipamento usado foi um FTIR Shimadzu, modelo 8300. Os espectros foram obtidos com resolução de $4 \mathrm{~cm}^{-1}$, com 100 varreduras. Para efeito de comparação também foi realizada uma análise termogravimétrica, usando-se um termo analisador Perkin Elmer. Essa análise foi feita em atmosfera de argônio com velocidade de aquecimento de $10{ }^{\circ} \mathrm{C} \mathrm{min}{ }^{-1}$.

\section{Resistência à lixiviação com solventes}

A metodologia utilizada no estudo da resistência à lixiviação com solventes foi de submeter $30 \mathrm{mg}$ de material em $5 \mathrm{~mL}$ de solvente, durante 12 minutos, sob ultra-som. Os solventes usados foram hexano e diclorometano, sendo ambos bidestilados. Após submeter ao ultrasom, o material foi centrifugado por 3 minutos, do qual foi extraído o sobrenadante, que foi concentrado, usando-se evaporação de arraste, com $\mathrm{N}_{2}$. A solução resultante foi analisada em um cromatógrafo gasoso, com detector seletivo de massas, modelo Shimadzu QP5050A. Usou-se uma coluna SPB-1 com $30 \mathrm{~m}$ x $25 \mathrm{~mm}$ x 0,25 $\mu \mathrm{m}$ de dimensões.

\section{RESULTADOS E DISCUSSÃO}

Foi obtido um gel de $p$-anisidinapropilsílica com área superficial de $93 \mathrm{~m}^{2} \mathrm{~g}^{-1}$ sendo que sua imagem obtida por MEV (Figura 1) mostrou a presença de aglomerados de partículas aproximadamente esféricas, com diâmetro médio de $184 \pm 42 \mathrm{~nm}$.

Os espectros no infravermelho, obtidos na temperatura ambiente, após o xerogel ter sido submetido a tratamento térmico, em várias temperaturas, são mostrados na Figura 2. A área sob a banda em $1511 \mathrm{~cm}^{-1}$, devida aos modos de deformação do anel aromático da panisidina, foi usada para estimar a estabilidade térmica desse grupo orgânico, nas diferentes temperaturas de tratamento (Tabela 1). Considerando-se possíveis heterogeneidades na espessura dos discos e também alterações na posição da amostra no feixe de infravermelho, as áreas das bandas da Tabela 1 foram calculadas usando-se a banda overtone da sílica, em $1870 \mathrm{~cm}^{-1}$, como banda de referência.

É possível observar na Tabela 1 e Figura 2 que a área sob a banda devida a $p$-anisidina é praticamente constante até a temperatura de $300{ }^{\circ} \mathrm{C}$, sob vácuo. Entre a temperatura de 100 e $300{ }^{\circ} \mathrm{C}$ tem-se uma redução na área de apenas $12 \%$. Essa excepcional estabilidade

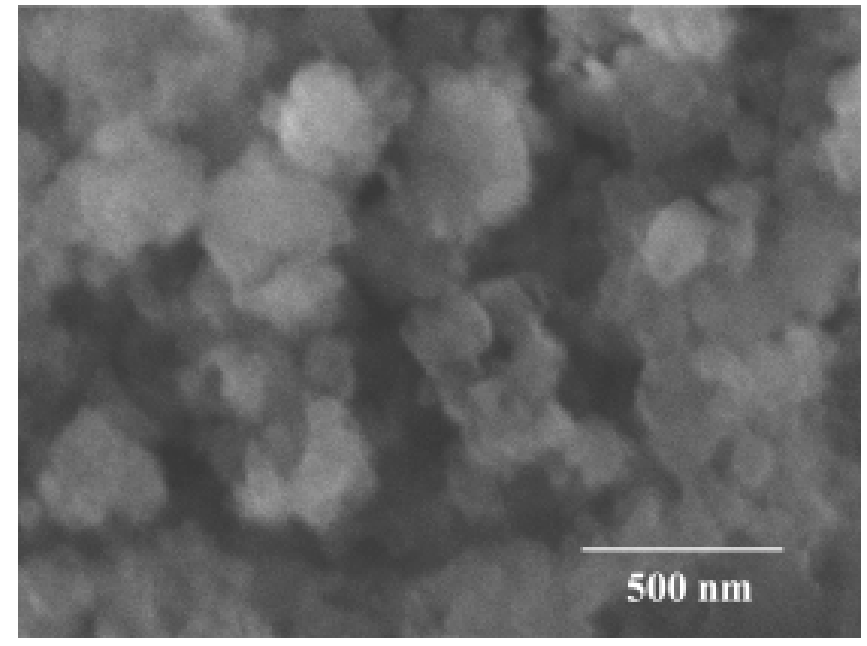

Figura 1. Imagem obtida por MEV ampliada 60000 vezes

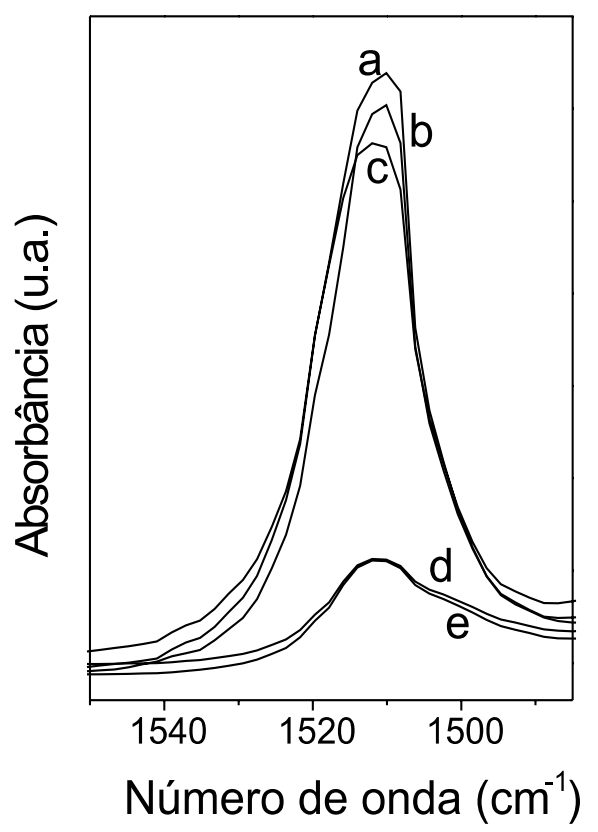

Figura 2. Espectros de absorção no infravermelho do xerogel panisidinapropilsilica, obtidos na temperatura ambiente, após o material ter sido submetido a tratamento térmico, sob vácuo. a) $100{ }^{\circ} \mathrm{C}$; b) $200{ }^{\circ} \mathrm{C}$; c) $300{ }^{\circ} \mathrm{C} ;$ d) $400{ }^{\circ} \mathrm{C}$; e) $450{ }^{\circ} \mathrm{C}$

térmica, em alto vácuo, é uma evidência de que esses grupos orgânicos estão fortemente ligados a sílica, na forma covalente. Para temperaturas superiores a $300{ }^{\circ} \mathrm{C}$ ocorreu uma drástica diminuição na área sob a banda da p-anisidina. $\mathrm{O}$ tratamento térmico a $400{ }^{\circ} \mathrm{C}$ deve 
Tabela 1. Área sob a banda no infravermelho em $1511 \mathrm{~cm}^{-1}$

\begin{tabular}{cc}
\hline Temperatura $\left({ }^{\circ} \mathrm{C}\right)$ & Área sob a banda ${ }^{\mathrm{a}}$ \\
\hline 100 & 6,2 \\
200 & 6,0 \\
300 & 5,5 \\
400 & 1,1 \\
450 & 1,1 \\
$100^{\mathrm{b}}$ & 5,6 \\
\hline
\end{tabular}

$\mathrm{a}=\frac{\left(\text { área sob a banda } 1511 \mathrm{~cm}^{-1}\right)}{\left(\text { área sob a banda } 1870 \mathrm{~cm}^{-1}\right)}$

${ }^{\mathrm{b}}=$ amostra exaustivamente lavada com diclorometano

ser suficientemente forte para produzir a dessorção dos grupos orgânicos da superfície. Entretanto mesmo após tratamento térmico a $450{ }^{\circ} \mathrm{C}$ ainda é possível observar no espectro a banda devida a panisidina. A presença de matéria orgânica mesmo após tratamento térmico a $450{ }^{\circ} \mathrm{C}$, sob vácuo, pode somente ser interpretada como sendo devida a uma fração de grupos orgânicos aprisionados em poros fechados. A fração de matéria orgânica oclusa foi estimada, a partir dos valores das áreas sob as bandas, como sendo de ca. $20 \%$.

A Figura 3 mostra o resultado da análise termogravimétrica onde até $150^{\circ} \mathrm{C}$ é observada a perda de massa típica, causada por evaporação de água e solventes adsorvidos na superfície do material. Entre 150 e $400{ }^{\circ} \mathrm{C}$ é observado uma região de massa aproximadamente constante. A pequena perda de massa, nessa região, é atribuída a reação de desidroxilação da superfície $i e$. conversão de grupos silanóis em siloxanos com liberação de água que é comum em xerogéis de sílica ${ }^{20}$. A partir de $400{ }^{\circ} \mathrm{C}$ ocorre uma perda considerável de massa que foi atribuída à eliminação dos grupos orgânicos que estavam incorporados ao sistema, juntamente com as reações de desidroxilação que continuam nessa faixa de temperatura.

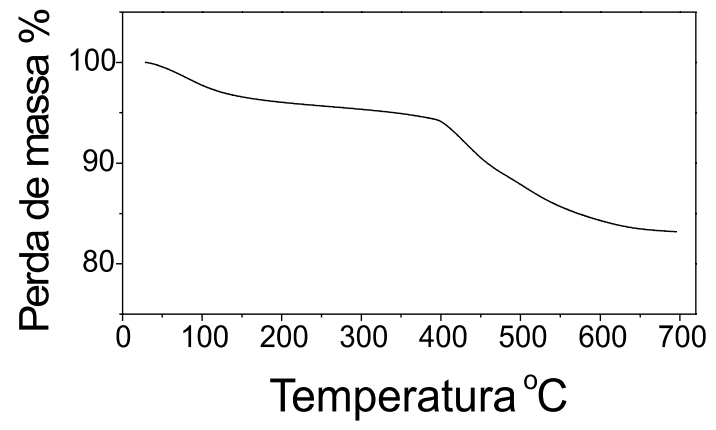

Figura 3. Gráfico de perda de massa em função da temperatura de aquecimento, obtido a partir da análise termogravimétrica do xerogel

As duas técnicas, espectroscopia no infravermelho e análise termogravimétrica mostraram concordância quanto a estabilidade térmica da fase orgânica. Entretanto, usando a espectroscopia no infravermelho a dessorção dos grupos orgânicos foi observada a partir de $300{ }^{\circ} \mathrm{C}$, enquanto que na análise termogravimétrica esse fenômeno somente foi detectado a partir de $400{ }^{\circ} \mathrm{C}$. Este fato é interpretado considerando-se as peculiaridades de cada técnica. Na análise termogravimétrica a amostra foi submetida a uma velocidade constante de aquecimento de $10{ }^{\circ} \mathrm{C} \mathrm{min}^{-1}$, enquanto que no infravermelho a amostra permanece durante 1 hora em cada temperatura de análise. Além disso, na análise no infravermelho a amostra é aquecida sob vácuo enquanto que na termogravimetria é usado pressão atmosférica.

Pode-se ressaltar que apenas na análise no infravermelho foi possível distinguir o comportamento térmico da fase orgânica, da desidroxilação da superfície da sílica, além de ter sido possível estimar a fração de grupos orgânicos aprisionados em poros fechados.

Para o estudo da resistência à lixiviação com solventes, o xerogel $p$-anisidinapropilsílica foi lavado inicialmente com hexano. $\mathrm{O}$ solvente de lavagem foi posteriormente submetido a análise cromatográfica. Os cromatogramas obtidos após sucessivas lavagens são mostrados na Figura 4. É possível observar nos cromatogramas um pico largo que foi identificado pelo espectrômetro de massas como sendo devido a hidrocarbonetos saturados lineares de grande cadeia $\left(\mathrm{C}_{20}\right.$ a $\left.\mathrm{C}_{35}\right)$. Certamente trata-se de resíduos da parafina presente no hidreto de sódio utilizado na reação de síntese do precursor AnPTMS. Entretanto o cromatograma do solvente, obtido após a terceira lavagem, é muito similar ao do hexano puro, indicando que a parafina foi extraída quase que completamente da superfície do xerogel após três sucessivas lavagens.

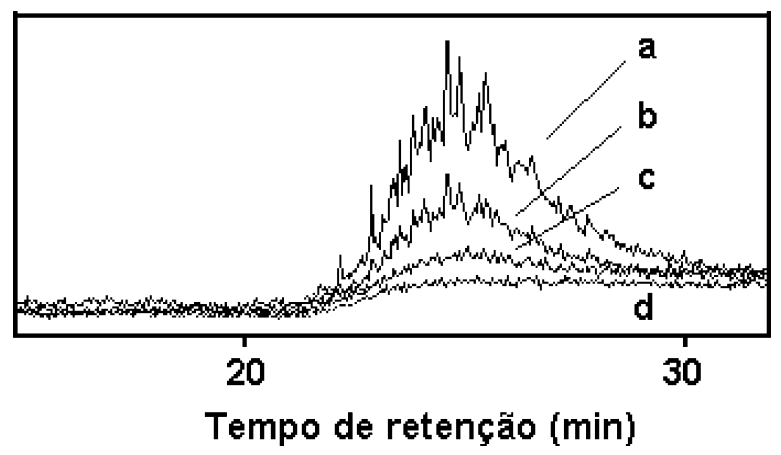

Figura 4. Cromatogramas do hexano de lavagem do xerogel panisidinapropilsilica a) primeira lavagem; b) segunda lavagem; c) terceira lavagem; d) hexano puro

Nas lavagens posteriores do xerogel com o solvente diclorometano, a análise cromatográfica não revelou picos, nem mesmo de hidrocarbonetos. Entretanto a cor do diclorometano da lavagem ficou violeta, muito semelhante a cor do xerogel. Esse resultado sugeriu que o diclorometano estaria lixiviando a fase orgânica. A fase lixiviada não foi detectada por cromatografia e nem mesmo no espectro de massas. Nesse caso o material lixiviado apresentaria um peso molecular maior que 500 u.m.a., com baixa volatilidade, sendo consequentemente retido no injetor (na pré coluna - liner), o que foi comprovado visualmente.

Para identificar a fase lixiviada, o diclorometano de lavagem foi evaporado e o resíduo violeta que sobrou foi depositado sobre um cristal de $\mathrm{NaCl}$ e analisado na região do infravermelho. $\mathrm{O}$ espectro no infravermelho do resíduo lixiviado é apresentado juntamente com o espectro do xerogel, na Figura 5. Pode se observar no espectro a da Figura 5, bandas de estiramento Si-O-Si, em $1100 \mathrm{~cm}^{-1} 20,21$ como também a banda devida a deformação do anel aromático da panisidina, em $1511 \mathrm{~cm}^{-1}$. O espectro do resíduo não corresponde portanto apenas a fase orgânica lixiviada, mas sim a uma fração de xerogel com alto grau de funcionalização orgânica. Possivelmente partículas muito pequenas que são arrastadas pelo solvente polar. $\mathrm{O}$ alto grau de funcionalização orgânica no resíduo é evidente quando comparamos os espectros a e b da Figura 5, onde a relação de área das bandas $1511 / 1100 \mathrm{~cm}^{-1}$ ou $1511 / 1870 \mathrm{~cm}^{-1}$ que correspondem a relação p-anisidina/sílica é claramente maior no resíduo do que no xerogel. No espectro do xerogel (Espectro b) a banda em 1100 é tão intensa, que ocorreu absorção total nessa região, enquanto que no espectro do resíduo é possível observar claramente os modos de estiramento característicos de $\mathrm{Si}-\mathrm{O}-\mathrm{Si}^{20,21}$.

Sucessivas lavagens do xerogel com diclorometano resultaram em descoloração do solvente de lavagem, indicando portanto que 


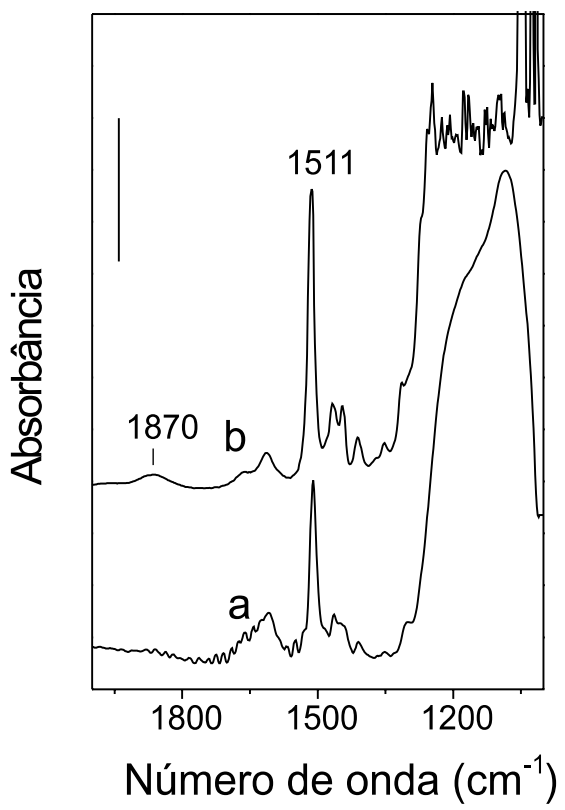

Figura 5. Espectros de absorção no infravermelho obtidos a temperatura ambiente. a) resíduo de lavagem com diclorometano; b) xerogel panisidinapropilsilica. $O$ valor da barra é 0,1 e 1,3 para os espectros a e b, respectivamente

após várias lavagens não há mais liberação de resíduo. Esse xerogel exaustivamente lavado com diclorometano foi então submetido a análise no infravermelho sob aquecimento a $100^{\circ} \mathrm{C}$, do qual foi calculado a área da banda em $1511 \mathrm{~cm}^{-1}$, que é devida a p-anisidina. $\mathrm{O}$ valor obtido está apresentado na Tabela 1 e foi semelhante aquele encontrado para o material submetido ao tratamento térmico a $300{ }^{\circ} \mathrm{C}$, ca. $10 \%$.

A semelhança nas áreas sob as bandas da p-anisidina nas amostras obtidas após aquecimento em alto vácuo e após lavagem exaustiva com diclorometano são um indicativo de que em ambos tratamentos há saída do mesmo resíduo, que foi caracterizado como uma sílica altamente funcionalizada, possivelmente partículas pequenas que não estão fortemente entrelaçadas à estrutura do xeroge ${ }^{22}$. O fato de que apenas o diclorometano consegue extrair esse resíduo pode ser interpretado considerando-se o maior momento dipolar desse solvente quando comparado ao hexano, até por que a p-anisidina também apresenta polaridade.

É importante ressaltar também que o xerogel previamente aquecido em vácuo a $300{ }^{\circ} \mathrm{C}$ não revelou presença de parafina frente a lavagem com hexano ou cor violeta após lavagem com diclorometano. Esse resultado é um indicativo de que a fração de xerogel altamente funcionalizada que é arrastada pelo diclorometano e a parafina residual podem também ser extraídas do xerogel através do tratamento térmico, em vácuo. Portanto o sólido resultante, lavado ou aquecido, apresentou apreciável estabilidade térmica e resistência à lixiviação com os solventes hexano e diclorometano.

\section{CONCLUSÕES}

O xerogel $p$-anisidinapropilsilica de dimensões nanométricas foi obtido. Esse xerogel apresenta, após sintetizado, resíduos de parafina que foi usada no processo da síntese como também apresenta uma pequena fração de sólido altamente funcionalizado que é arrastado pelo diclorometano. Sucessivas lavagens com hexano e diclorometano, são suficientes para produzir a limpeza do xerogel. Alternativamente o xerogel pode ser purificado através do tratamento térmico na temperatura de $300{ }^{\circ} \mathrm{C}$, sob vácuo. Assim podemos inferir que o xerogel purificado apresenta boa estabilidade térmica e resistência à lixiviação com os solventes hexano e diclorometano, que são características importantes para materiais potencialmente aplicáveis, como fases estacionárias, em processos de separação.

\section{REFERÊNCIAS}

1. Deschler, U.; Kleinschmit, P.; Panster, P.; Angew. Chem., Int. Ed. 1986, $25,236$.

2. Shintani, H. J.; Liquid Chromat. 1992, 15, 8 .

3. Kiseleva, M. G.; Nesterenko, P. N.; J. Chromatogr. A 2000, 898, 23.

4. Arakaki, N. H.; Airoldi, C.; Quim. Nova 1999, 22, 246.

5. Silberzan, P.; Léger, L.; Ausserré, D.; Benattar, J. J.; Langmuir 1991, 7, 1647.

6. Gambero, A.; Kubota, L. T.; Gushikem, Y.; Airoldi, C.; Granjeiro, J. M.; Taga, E. M.; Alcantara, E. F. C.; J. Colloid Interface Sci. 1997, 185, 313.

7. Hench, L. L.; West, J. K.; Chem. Rev. 1990, 90, 33

8. Loy, D. A.; Shea, K. J.; Chem. Rev. 1995, 95,1431.

9. Hiratsuka, R. S.; Santilli,C. V.; Pulcinelli, S. H.; Quim. Nova 1995, 18, 171.

10. Sacco, H. C.; Ciuffi, K. J.; Biazzotto, J. C.; Zuccki, M. R.; Leite, C. A. P.; Nascimento, O. R.; Serra, O. A.; Iamamoto, Y.; J. Non-Cryst. Solids 2000, 273, 150.

11. Pavan, F. A.; Leal, S.; Costa, T. M. H.; Benvenutti, E. V.; Gushikem, Y.; J. Sol-Gel Sci. Technol. 2002, 23, 129.

12. Lev, O.; Tsionsky, M.; Rabinovich, L.; Glazer, V.; Sampath, S.; Pankratov, I.; Gun, J.; Anal. Chem. 1995, 67, 22A.

13. Corriu, R. J. P.; Leclercq, D.; Angew. Chem., Int. Ed. 1996, 35, 5.

14. C. Brinker, J.; Scherer, G. W.; Sol-Gel Science, Academic Press: London, 1990.

15. Collinson, M. M.; Crit. Rev. Anal. Chem. 1999, 29, 289.

16. Pavan, F. A.; Franken, L.; Moreira, C. A.; Costa, T. M. H.; Benvenutti, E. V.; Gushikem Y. ; J. Colloid Interface Sci. 2001, 241, 413.

17. Bernards, T. N. M.; Van Bommel, M. J.; Jansen, J. A. J.; J. Sol-Gel Sci. Technol. 1998, 13, 749.

18. Pinto, L. C. M.; Tese de Doutorado, Universidade de São Paulo, Brasil, 1996.

19. Foschiera, J. L.; Pizzolato, T. M.; Benvenutti, E. V.; J. Braz. Chem. Soc. 2001, 12, 159

20. Costa, T. M. H.; Gallas, M. R.; Benvenutti, E. V.; da Jornada, J. A. H.; J. Non-Cryst. Solids 1997, 220, 195

21. Wood, D. L.; Rabinovich, E. M.; Appl. Spectrosc. 1989, 43, 263.

22. Schubert, U.; Hüsing, N.; Lorenz, A.; Chem. Mater. 1995, 7, 2010. 